\title{
Tripod Turnstile Machines Performance Analysis for the System Safety and Security without Considering Simultaneous Failures using Reliability Approach
}

\author{
Pardeep Kumar \\ Department of Mathematics, Faculty of Science, \\ Lovely Professional University, Phagwara, Punjab, India. \\ E-mail: pardeep.20221@lpu.co.in \\ Amit Kumar \\ Department of Mathematics, Faculty of Science, \\ Lovely Professional University, Phagwara, Punjab, India. \\ Corresponding author: amit.20445@1pu.co.in, amit303singh@gmail.com
}

(Received May 21, 2020; Accepted August 23, 2020)

\begin{abstract}
As the crime is increasing day by day around the world. To stop the crime many security gadgets and machines have been developed by various security agencies. The tripod turnstile machine also helps in stopping the crime and restricting the entry of unauthorized person on the premises. In this paper, the aim is to analyze the reliability measures of the two tripod turnstile machines which work in a parallel configuration. In the case of non-functionality of the machine, unauthorized persons may enter into the premises. Due to security and safety reason, organizations install these machines. Hence these machines must be highly reliable in the operation to avoid any unwanted event. In this paper, for the considered system, the transition state diagram has been drawn with the help of the Markov model. The ChapmanKolmogorov differential equations are developed from the transition state diagram and solved using Laplace transformation. The failure and repair rates are assumed to be constant and follow negative exponential distribution. The system upstate and downstate probabilities are determined. The explicit expression of the system Availability, Reliability and MTTF are also obtained. The sensitivity analysis is also performed to determine which machine affects the system reliability the most.
\end{abstract}

Keywords- Tripod turnstile machine, Markov model, Laplace transformation, MTTF, Reliability, Availability.

\section{Introduction}

In the whole world, crime is increasing day by day. Therefore, human safety and system safety are the main concerns of the society. With the advancement in the field of science and technology, many useful gadgets and machines have been developed to stop the crime. Though the crime has not been fully eradicated, these gadgets and machines help in the reduction of the rate of crime. The turnstile machine also plays a very crucial role to restrict the entry of the unauthorized person. For this reason, turnstile machines are generally installed at metro railway stations, airports and stadium etc. Turnstile machine is also known as baffle gate. It allows only one person to pass when a person punches a card or insert a coin in the machine. From a security point of view, this is very important to install a turnstile machine as it also stops thefts and crime in the organization. When a person punches his card on the card reader this machine stores the data like name, UID, time of entry, time of exit. Hence, it helps to reduce the security concern of the people. For this reason, failure-free operation of the turnstile machines is required. These turnstile machines come in different sizes like waist height, full- height turnstile, tripod, flap gates etc. Per-minute 15-20 people can pass from this gate using their card or by inserting a coin in the machine. Mosman et al. (2019) 
International Journal of Mathematical, Engineering and Management Sciences

Vol. 6, No. 1, 383-395, 2021

https://doi.org/10.33889/IJMEMS.2021.6.1.024

introduced the concept of automated stadium management system for managing the crowd of a football stadium. With this system, the data of football match viewer, present in the stadium can be easily saved. With this information, if any unwanted event happens, then the investigation can be done easily with the help of the data available.

On the failure of the turnstile machine, it is either repaired or replaced with a new machine, hence, it is a repairable system. A repairable system is one which has two states, perfect working and complete failure. The repairable system always oscillates between these two states. When the system fails, out of repair or replacement, repair of the system is always preferred. Replacement of the system or component is the best option when the repair is very costly. There are basically two types of maintenance policies, Corrective maintenance and preventive maintenance. Corrective maintenance is performed on the failure of the system and preventive maintenance is performed to delay the failure of the system. Literature related to maintenance policies can be found in McCall (1965), Pierskalla and Voelker (1976), Cho and Parlar (1991), Sherif and Smith (1981), Kumar and Ram (2016). Maintenance action is performed on the system or on system components. Basically, a system is a collection of components or machines that are connected to perform a specific task. A system may be in a series configuration, parallel configuration or in a mixed configuration. Description of all these can be easily found in Srinath (1994), Balagurusamy (1984), Ebeling (2004), Ram et al. (2013), Ram and Kumar (2015), Kumar et al. (2017). Besides, these configurations, redundancy also plays a very important role in increasing system reliability and availability. There are basically two types of redundancies active redundancy and standby redundancy. Li (2016) presented that standby redundancy is better than the active redundancy. Zheng et al. (2018) investigates a real time computing system by the aid of continuous time Markov chain and a comparison was done in between effect of CCFs on the system in warm and hot standby configurations.

To determine the reliability measures of the system, Markov modelling is being used extensively these days by the researchers. The Markov-model is a state-based model. The main advantage of the Markov model is the transition of the future states depend only on the present state but not on the past states. Kumar and Kumar (2019a) used the Markov model for the modelling of the wireless communication system and various reliability measures were calculated in their paper. Authors Kumar and Kumar (2019b), Niwas and Garg (2018) investigated an industrial system which works under cost-free warranty policy. They used the Markov model to determine the reliability indices of the system.

Sharma and Vishwakarma (2014) further extended the work and analyzed the performance of the feeding system of the sugar industry. Using Genetic algorithm steady state availability of the system was optimized. Yusuf et al. (2018) analyzed the performance of single host with three types of software. Initially, one software is used but when it fails other software of the same type is used. In this way failure of software doesn't cause system to fail on the failure of the software. This helps in extending the availability of the system and increasing the revenue of the system. Numerical method techniques have also been used in the literature to determine the reliability of the industrial system. Shakuntla et al. (2011) determined the reliability of the polytube industry using by using Runge-Kutta fourth order method. Sensitivity analysis was also carried out to improve the performance of availability. This discussion gives us the idea of the wide application of the Markov model in industries. 
International Journal of Mathematical, Engineering and Management Sciences

Vol. 6, No. 1, 383-395, 2021

https://doi.org/10.33889/IJMEMS.2021.6.1.024

To the best of the authors' knowledge, there is not much literature available for the turnstile machines and no one has ever tried to determine the reliability measures of the turnstile machine. In this paper, authors aim is to determine the reliability measure of the turnstile machines working in a series configuration.

\subsection{Problem Statement}

It has been observed, these turnstile machines go out of order many a time when their failure-free operation is required. This allows an unauthorized person to enter into the premises. The entry of these unauthorized persons may affect human security and system safety sometimes. Hence keeping this problem in the mind, reliability measures of the turnstile machine are determined in this paper. This paper is organized as follows: In section (2), system description, assumptions and nomenclature are given. In section (3), Chapman-Kolmogorov differential equations are developed from the transition state diagram. In section (4), for the considered system Availability, Reliability and MTTF are calculated. Besides this, Sensitivity analysis is also performed. In section (5) results are presented. In section (6), Conclusion is given. In section (7), future scope of the work is given. In section (8), conflict of interest is also given.

\section{System Description}

In this paper, authors consider two turnstile machine, these machines are working in a parallel configuration. Initially, both the machines are in good working condition. When one turnstile machine fails, then other turnstile machine continues to work and meanwhile, the failed machine is repaired. After repair the turnstile machine is as good as the new machine. On the failure of both machines the entire security system fails. As only one repairman is available with the organization, therefore, the repair of the machine is done on first come and first-served basis. Repair and the failure rates are assumed to be constant. Later in the paper, reliability measures have been determined by substituting the particular value of the repair and failure rates in the system upstate probability.

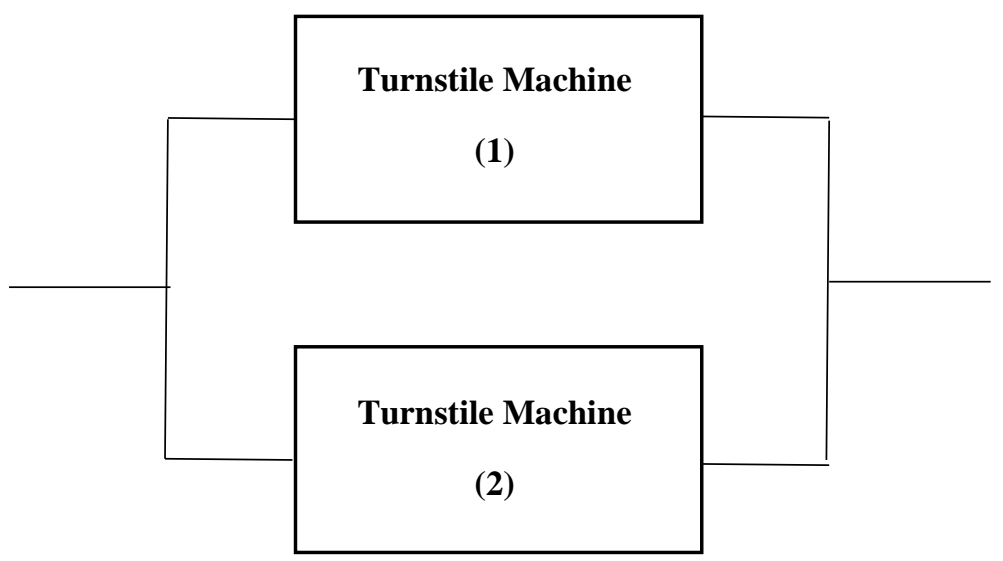

Figure 1. Reliability block diagram of turnstile machines. 
International Journal of Mathematical, Engineering and Management Sciences

Vol. 6, No. 1, 383-395, 2021

https://doi.org/10.33889/IJMEMS.2021.6.1.024

\subsection{Assumption of the System}

(i) Initially, both the turnstile machines are in good working condition.

(ii) Failure rates of the machine are statistically independent.

(iii) Sufficient repair facility is available.

(iv) After repair, the system is as good as a new one.

(v) Repair and failure rates are assumed to be constant.

(vi) Both machines cannot fail simultaneously.

(vii) Repair of the machine is performed on a first come first-served basis.

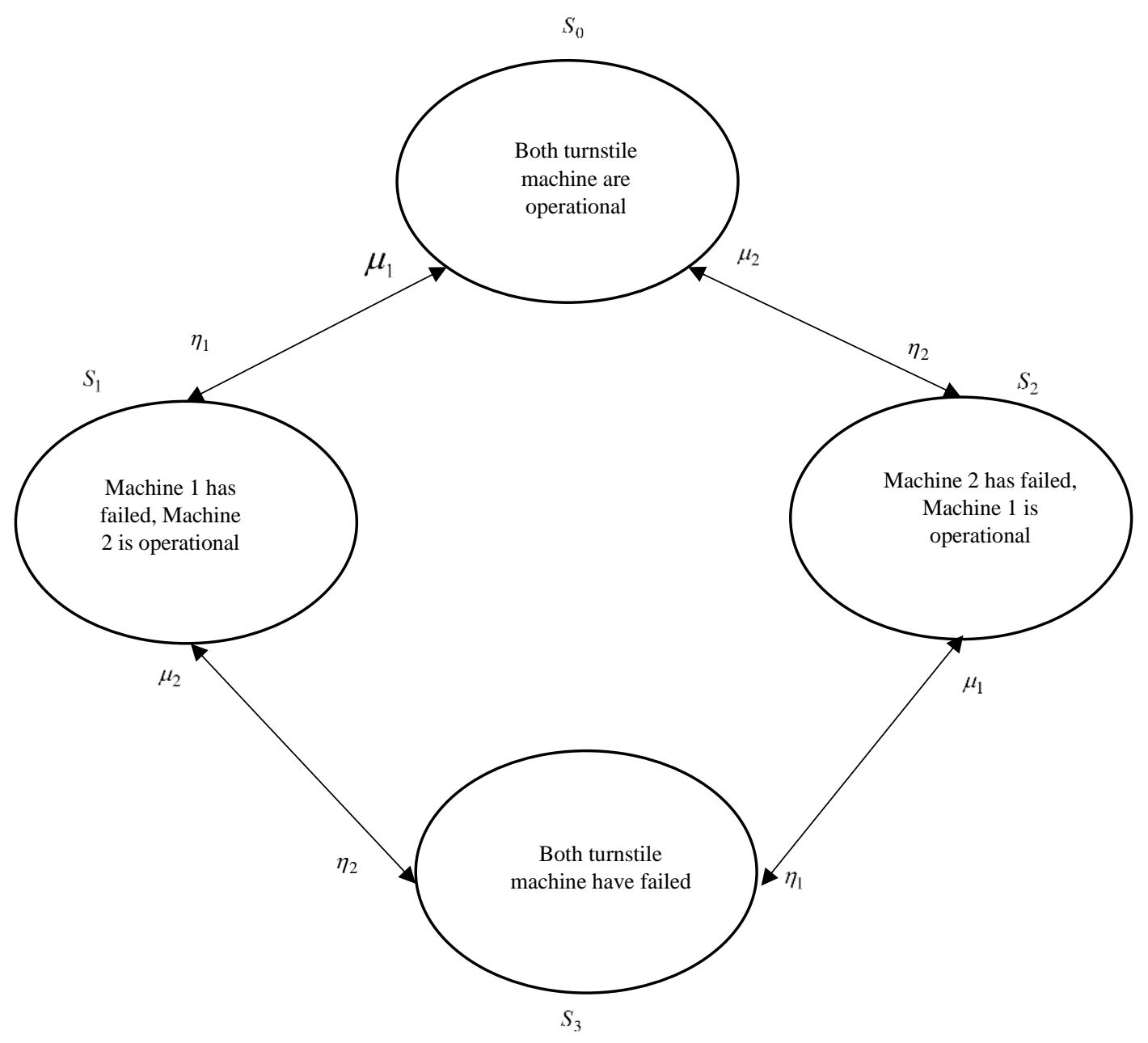

Figure 2. State transition diagram of turnstile machines. 
International Journal of Mathematical, Engineering and Management Sciences

Vol. 6, No. 1, 383-395, 2021

https://doi.org/10.33889/IJMEMS.2021.6.1.024

\subsection{Nomenclature}

The nomenclature of the work are found in Table 1.

Table 1. Notations.

\begin{tabular}{|l|l|}
\hline Notation & Description \\
\hline$t$ & Time variable \\
\hline$S$ & Laplace transform variable \\
\hline$P_{i}(t) ; i=0,1,2,3$ & Probability that system at time $t$ being in the state $S_{i}(t)$ \\
\hline$\overline{P_{i}}(s) ; i=0,1,2,3$ & Laplace transform of $P_{i}(t)$ \\
\hline$\eta_{1} / \eta_{2}$ & Failure rate of the first/second turnstile machine \\
\hline$\mu_{1} / \mu_{2}$ & Repair rate of the first/second turnstile machine \\
\hline$S_{i} ; i=0,1,2,3$ & States of the system \\
\hline
\end{tabular}

\section{Mathematical Formulation of the Turnstile System}

In order to determine the reliability measures of the system, as it is presented in Figure 1, the transition state diagram of the system is drawn as shown in Figure 2, Authors has been developed the following Chapman-Kolmogorov differential equations from the transition state diagram of the system given in Figure 2.

$\left[\frac{d}{d t}+\eta_{1}+\eta_{2}\right] P_{0}(t)=\mu_{1} P_{1}(t)+\mu_{2} P_{2}(t)$

$\left[\frac{d}{d t}+\mu_{1}+\eta_{2}\right] P_{1}(t)=\mu_{2} P_{3}(t)+\eta_{1} P_{0}(t)$

$\left[\frac{d}{d t}+\mu_{2}+\eta_{1}\right] P_{2}(t)=\mu_{1} P_{3}(t)+\eta_{2} P_{0}(t)$

$\left[\frac{d}{d t}+\mu_{2}+\mu_{1}\right] P_{3}(t)=\eta_{2} P_{1}(t)+\eta_{1} P_{2}(t)$

With initial condition;

$P_{i}(0)= \begin{cases}1 & i=0 \\ 0 & i \neq 0\end{cases}$

Taking Laplace transformation of equations from (1) to (4), we get;

$$
\begin{aligned}
& {\left[s+\eta_{1}+\eta_{2}\right] \overline{P_{0}}(s)=1+\mu_{1} \overline{P_{1}}(s)+\mu_{2} \overline{P_{2}}(s)} \\
& {\left[s+\mu_{1}+\eta_{2}\right] \overline{P_{1}}(s)=\mu_{2} \overline{P_{3}}(s)+\eta_{1} \overline{P_{0}}(s)} \\
& {\left[s+\mu_{2}+\eta_{1}\right] \overline{P_{2}}(s)=\mu_{1} \overline{P_{3}}(s)+\eta_{2} \overline{P_{0}}(s)}
\end{aligned}
$$


International Journal of Mathematical, Engineering and Management Sciences

Vol. 6, No. 1, 383-395, 2021

https://doi.org/10.33889/IJMEMS.2021.6.1.024

$\left[s+\mu_{1}+\mu_{2}\right] \overline{P_{3}}(s)=\eta_{2} \overline{P_{1}}(s)+\eta_{1} \overline{P_{2}}(s)$

On solving equations from (6) - (9), we get;

$\overline{P_{0}}(s)=\frac{1}{\left[H_{1}-\mu_{1} K_{1}-\mu_{2} K_{2}\right]}$

$\overline{P_{1}}(s)=K_{1} \overline{P_{0}}(s)$

$\overline{P_{2}}(s)=K_{2} \overline{P_{0}}(s)$

$\overline{P_{3}}(s)=\left[\frac{\overline{P_{0}}(s) H_{5}}{H_{6}}\right]$

where,

$$
\begin{aligned}
& H_{1}=\left[s+\eta_{1}+\eta_{2}\right], H_{2}=\left[s+\mu_{1}+\eta_{2}\right], H_{3}=\left[s+\mu_{2}+\eta_{1}\right], H_{4}=\left[s+\mu_{1}+\mu_{2}\right], \\
& H_{6}=\left[1-\frac{\eta_{2} \mu_{2}}{H_{2} H_{4}}-\frac{\eta_{1} \mu_{1}}{H_{3} H_{4}}\right], K_{1}=\left[\frac{\eta_{1}}{H_{2}}+\frac{\mu_{2} H_{5}}{H_{2} H_{6}}\right], K_{2}=\left[\frac{\eta_{2}}{H_{3}}+\frac{\mu_{1} H_{5}}{H_{3} H_{6}}\right] .
\end{aligned}
$$

Therefore, the system upstate (good and degraded states) and downstate (failed states) are given by:

$$
\begin{aligned}
& P_{u p}(s)=\overline{P_{0}}(s)+\overline{P_{1}}(s)+\overline{P_{2}}(s) \\
& P_{d o w n}(s)=\overline{P_{3}}(s)
\end{aligned}
$$

\section{Numerical Computation}

The following values of failure and repair rates will be used for the calculation in this paper.

Table 2. Failure and repair rate of Turnstile machines.

\begin{tabular}{|c|c|c|}
\hline Component & Failure rate/per hour & Repair rate/per hour \\
\hline Turnstile machine 1 & $\eta_{1}=0.0000100$ & $\mu_{1}=0.0066666$ \\
\hline Turnstile machine 2 & $\eta_{2}=0.0000125$ & $\mu_{2}=0.0050000$ \\
\hline
\end{tabular}

\subsection{Availability of the System}

Availability of a repairable system is the probability that the system is performing its task at a specified period of time under given operating condition. For calculating the system availability of 
International Journal of Mathematical, Engineering and Management Sciences

Vol. 6, No. 1, 383-395, 2021

https://doi.org/10.33889/IJMEMS.2021.6.1.024

the proposed system, take inverse Laplace of equation (14) and substitute the values of failure rates and repair rates given in Table 2, one can easily get the explicit expression of the system availability.

$$
\begin{aligned}
A(t)= & 0.000003735418793 e^{-0.0066760 t}+0.000003735418793 e^{-0.0050125 t}+0.9999962646 \\
& -0.000003735418793 e^{-0.01168850 t}
\end{aligned}
$$

On varying time unit from 0 to 100000 with the step size of 10000 , one can easily analyze the behavior of system availability from Table 3 and Figure 3.

Table 3. Availability of the system.

\begin{tabular}{|c|c|}
\hline Time (In hrs.) & Availability \\
\hline 0 & 1.00000 \\
\hline 10000 & 0.99999 \\
\hline 20000 & 0.99999 \\
\hline 30000 & 0.99999 \\
\hline 40000 & 0.99999 \\
\hline 50000 & 0.99999 \\
\hline 60000 & 0.99999 \\
\hline 70000 & 0.99999 \\
\hline 80000 & 0.99999 \\
\hline 90000 & 0.99999 \\
\hline 100000 & 0.99999 \\
\hline
\end{tabular}

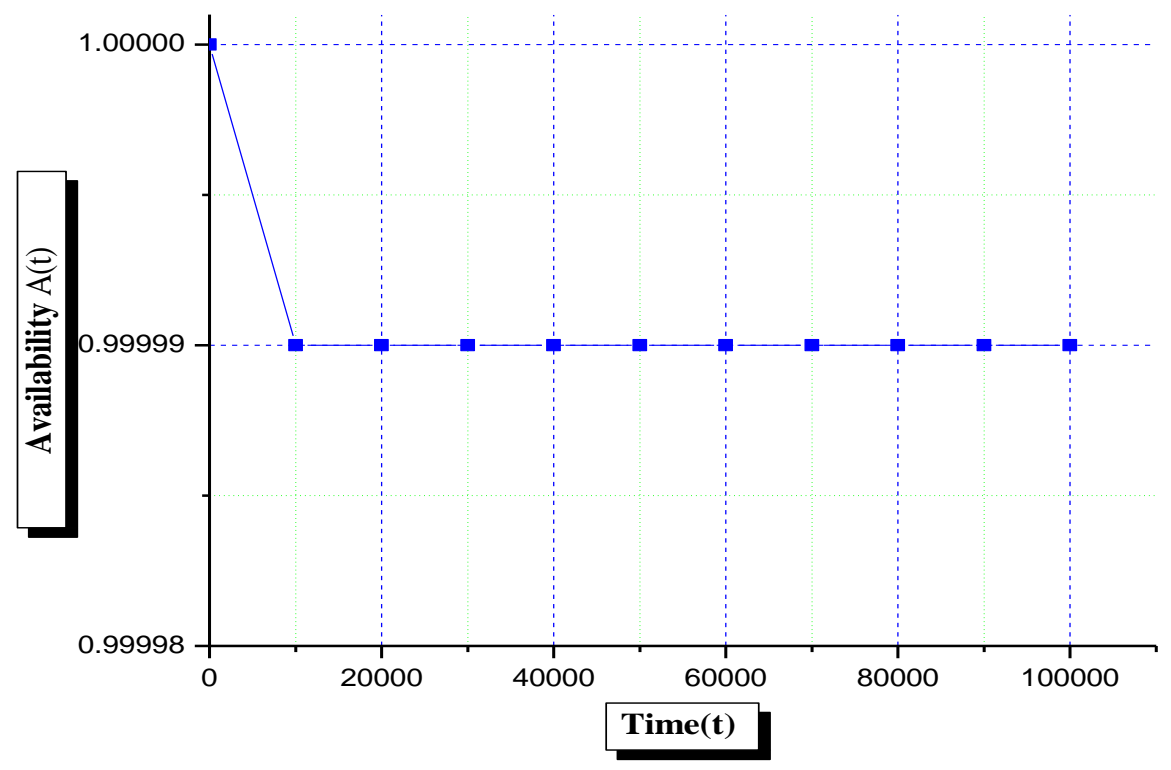

Figure 3. Availability of the system w.r.t. time. 
International Journal of Mathematical, Engineering and Management Sciences

Vol. 6, No. 1, 383-395, 2021

https://doi.org/10.33889/IJMEMS.2021.6.1.024

\subsection{Reliability of the System}

Reliability is the quantitate attribute of the system. Generally, reliability is the probability that the system will perform its task as expected without failure for a specified period of time under specified conditions. If the system does not perform in the specified conditions then it is said to be the failure of the system. For the proposed system, one can easily get the explicit expression for reliability. For this, take Inverse Laplace of the equation (14). After this, substitute the values of failure rates as given in Table 2 and also set the repair rates equal to zero in the obtained expression. One can easily get the explicit expression of the reliability of the system.

$R(t)=e^{-0.0000225 t}+2 e^{-0.0000175 t} \sinh (0.000005 t)+2 e^{-0.00001625 t} \sinh (0.00000625 t)$

On varying time unit from 0 to 100000 with the step size of 10000 , one can easily analyze the behavior of system reliability from Table 4 and Figure 4.

Table 4. Reliability of the system.

\begin{tabular}{|c|c|}
\hline Time (In hrs.) & Reliability \\
\hline 0 & 1 \\
\hline 10000 & 0.98881 \\
\hline 20000 & 0.95990 \\
\hline 30000 & 0.91895 \\
\hline 40000 & 0.87028 \\
\hline 50000 & 0.81713 \\
\hline 60000 & 0.76193 \\
\hline 70000 & 0.70643 \\
\hline 80000 & 0.65190 \\
\hline 90000 & 0.59922 \\
\hline 100000 & 0.54898 \\
\hline
\end{tabular}

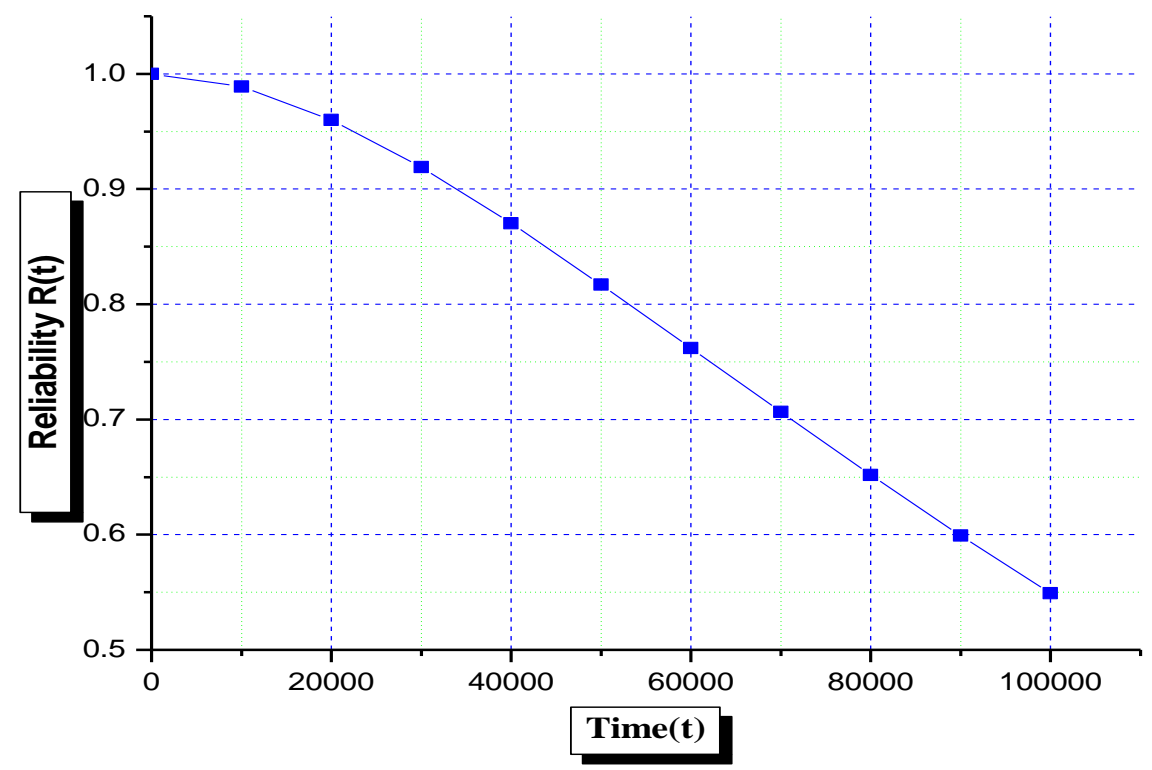

Figure 4. Reliability of the system w.r.t. time. 
International Journal of Mathematical, Engineering and Management Sciences

Vol. 6, No. 1, 383-395, 2021

https://doi.org/10.33889/IJMEMS.2021.6.1.024

\subsection{MTTF (Mean Time to Failure)}

Mean time to failure is the expected time for the system failure. To find the mean time to failure set all repair rates equal to zero in (14), and then taking the limit $s \rightarrow 0$, one can easily get the MTTF of the system. The formula for obtaining the MTTF is given below

$M T T F=\int_{0}^{\infty} R(t) d t=\lim _{s \rightarrow 0} R(s)$

The explicit expression of the MTTF is

$M T T F=\frac{\eta_{1}^{2}+2 \eta_{1} \eta_{2}+\eta_{2}^{2}}{\left(\eta_{1}+\eta_{2}\right) \eta_{1} \eta_{2}}$

Setting values of failure rates as given in Table 2 and varying each failure rate one by one from 0.00001 to 0.00010 in (19), one can get the variation in the MTTF w.r.t variation in the failure rates. This can be easily analyzed from Table 5 and Figure 5.

Table 5. MTTF of the system.

\begin{tabular}{|c|c|c|}
\hline Variation in failure rates & MTTF with respect to $\eta_{1}$ & MTTF with respect to $\eta_{2}$ \\
\hline 0.00001 & 135555.56 & 150000.00 \\
\hline 0.00002 & 99230.76 & 116666.67 \\
\hline 0.00003 & 89803.92 & 108333.33 \\
\hline 0.00004 & 85952.38 & 105000.00 \\
\hline 0.00005 & 84000.00 & 103333.33 \\
\hline 0.00006 & 82873.56 & 102380.95 \\
\hline 0.00007 & 82164.50 & 101785.71 \\
\hline 0.00008 & 81689.18 & 101388.89 \\
\hline 0.00009 & 81355.01 & 101111.11 \\
\hline 0.00010 & 8111.11 & 100909.09 \\
\hline
\end{tabular}

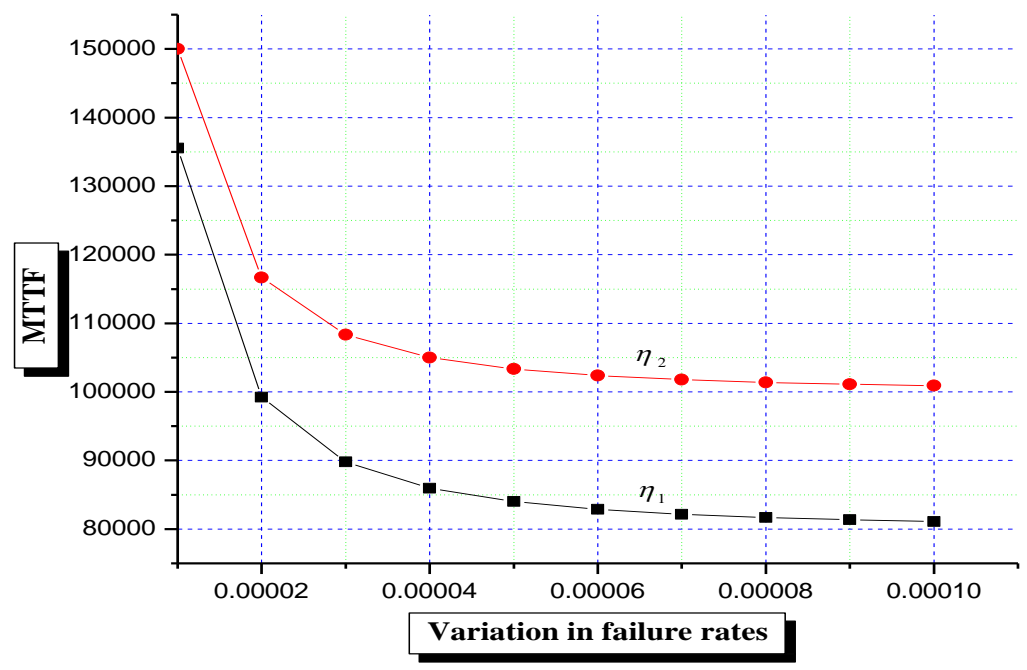

Figure 5. MTTF W.r.t. variation in failure rates. 
International Journal of Mathematical, Engineering and Management Sciences

Vol. 6, No. 1, 383-395, 2021

https://doi.org/10.33889/IJMEMS.2021.6.1.024

\subsection{Sensitivity of Reliability}

Sensitivity analysis is performed to determine the most critical component/ components of the system. It actually determines, how actually the system performance measure is affected by changing the failure rate of the system's component. Here, authors perform the sensitivity analysis of the reliability of the system. For this first of all take the inverse Laplace of (14) and differentiate the obtained expression w.r.t all the failure rates one by one. In these derivatives set failure rates values as given in Table 2 and also set all repair rate equal to zero. On varying the time unit from 0 to 100000 with the step size of 10000, one can easily obtain Table 6 and Figure 6 .

Table 6. Sensitivity of reliability.

\begin{tabular}{|c|c|c|}
\hline Time (In hrs.) & $\frac{\partial(R(t))}{\partial \eta_{1}}$ & $\frac{\partial(R(t))}{\partial \eta_{2}}$ \\
\hline 0 & 0 & 0 \\
\hline 10000 & -1063.21 & -839.80 \\
\hline 20000 & -3622.05 & -2823.45 \\
\hline 30000 & -6949.85 & -5343.98 \\
\hline 40000 & -10550.01 & -7998.44 \\
\hline 50000 & -14093.90 & -10530.44 \\
\hline 60000 & -17374.28 & -12787.57 \\
\hline 70000 & -20270.44 & -14689.81 \\
\hline 80000 & -22722.40 & -16206.44 \\
\hline 90000 & -24711.82 & -17339.27 \\
\hline 100000 & -26248.02 & -18110.55 \\
\hline
\end{tabular}

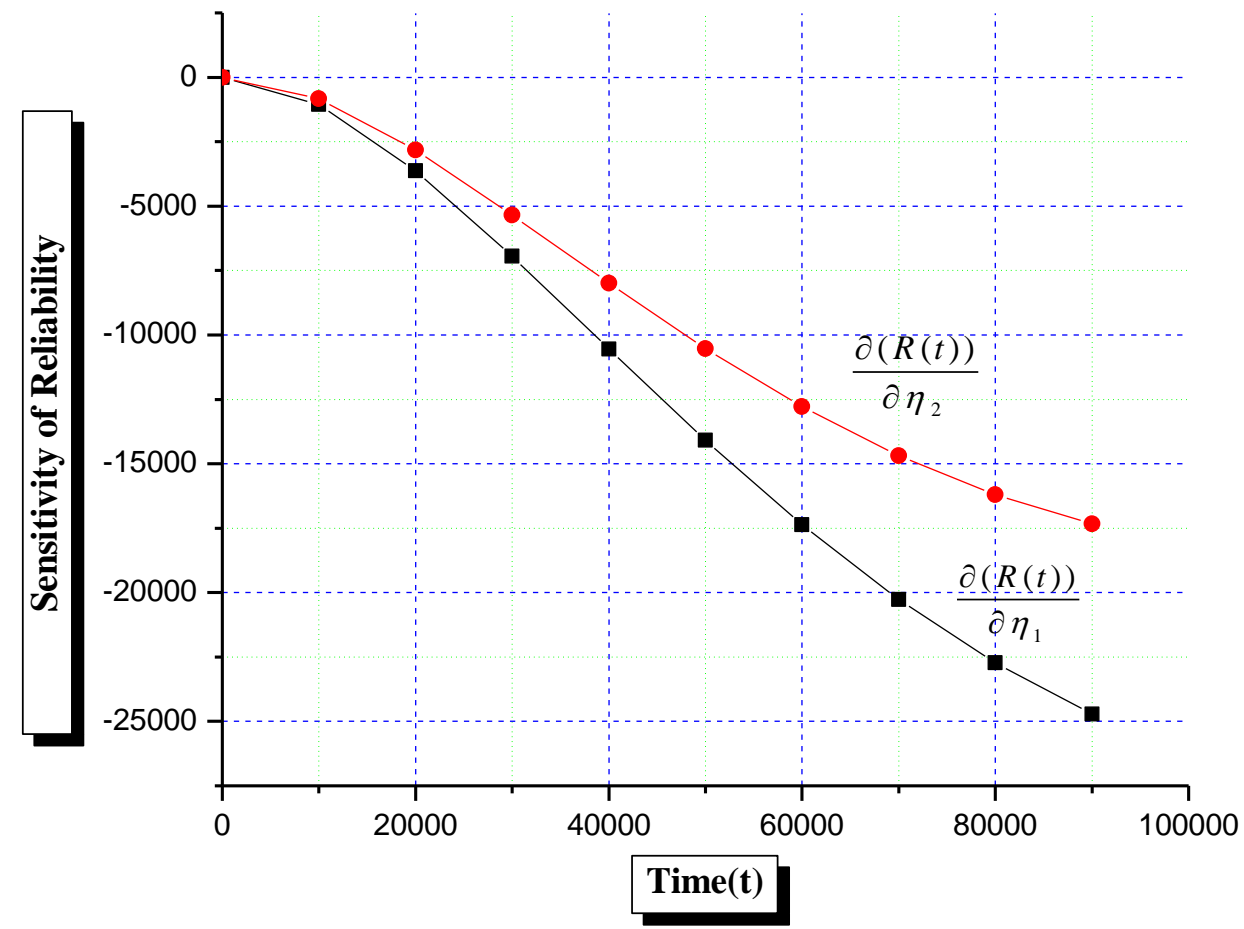

Figure 6. Sensitivity of reliability w.r.t. time. 
International Journal of Mathematical, Engineering and Management Sciences

Vol. 6, No. 1, 383-395, 2021

https://doi.org/10.33889/IJMEMS.2021.6.1.024

\section{Result Discussion}

In this paper, a system of two turnstile machines which work in a parallel configuration have been considered. The various reliability measures like availability, reliability and MTTF have been evaluated. Sensitivity analysis of the reliability is also performed. From the above Tables and Graphs following results are obtained.

(i) From Table 3 and Figure 3, one can easily see that system availability is very high. After 100000 hours of operation, system availability is .99999 . This availability graph is constant for the time period 10000 to 100000 . For 90000 hours the system availability remains the same.

(ii) From Table 4 and Figure 4, one can easily see that the reliability of the system decreases as the time ' $t$ ' increases in a smooth manner. After 100000 hours of operation, system reliability is 0.54898 .

(iii) From Table 5 and Figure 5, one can see that as the failure rate increases of both the turnstile machines, the MTTF of the system decreases continuously. System MTTF is high, in case of, the variation in the failure rate of the second tripod turnstile machine as compared to the MTTF of the first tripod turnstile machine.

(iv) From Table 6 and Figure 6, one can see that system reliability is more affected by the variation in the failure rate of the first tripod turnstile machine.

\section{Conclusion}

Finally, we conclude on the basis of the above result discussion section, these machines are highly available, reliable and secure for the safety of the organization. In a secure and safe environment, the productivity of each person improves. If the organization wants, then more attention must be given to improve the performance of the first turnstile machine, as the reliability of the system is very much affected by the variation in the failure rate of the first tripod machine. The engineer must timely plan the predictive maintenance of the turnstile machine so that less failure occur during the rush hours. Authors, of this paper, are hopeful this research will be quite useful for the tripod turnstile machine companies and for those organizations which install these machines in their premises.

Also, at the future point of view (i) reliability measures can also be determined by considering a multi-state turnstile system as the performance rate of the machine may vary for the different organizations. (ii) at some places these machines are installed in the open area. Reliability measures of the machine can be determined under the effect of natural phenomenon like rain, storm etc. (iii) the work can be extended by finding the busy period of the repairman, MTBF, profit analysis of the system etc.

\section{Conflict of Interest}

The authors declared that there is no conflict of interest.

\section{Acknowledgements}

The authors are highly thankful to "HONESTY AUTOMATION PVT. LTD" for providing support to carry out this research work. Also, authors are very thankful to the editor and reviewers for their valuable suggestion for the improvement of the paper. 
International Journal of Mathematical, Engineering and Management Sciences

Vol. 6, No. 1, 383-395, 2021

https://doi.org/10.33889/IJMEMS.2021.6.1.024

\section{References}

Balagurusamy, E. (1984). Reliability engineering. Tata McGraw-Hill Education, New Delhi, India.

Cho, D.I., \& Parlar, M. (1991). A survey of maintenance models for multi-unit systems. European Journal of Operational Research, 51(1), 1-23.

Ebeling, C.E. (2004). An introduction to reliability and maintainability engineering. Tata McGraw-Hill Education, New York.

Kumar, A., \& Kumar, P. (2019a). Application of Markov process/mathematical modelling in analysing communication system reliability. International Journal of Quality \& Reliability Management, 37(2), 354-371.

Kumar, A., \& Ram, M. (2016). Performance analysis of pulping and screening system of a paper production plant. Neural, Parallel, and Scientific Computations, 24, 351-368.

Kumar, A., Ram, M., \& Rawat, R.S. (2017). Optimization of casting process through reliability approach. International Journal of Quality \& Reliability Management, 34(6), 833-848.

Kumar, P., \& Kumar, A. (2019b). Reliability analysis of an industrial system under cost-free warranty and system rest policy. Journal of KONBiN, 49(2), 377-395.

Li, J. (2016). Reliability comparative evaluation of active redundancy vs. standby redundancy. International Journal of Mathematics Engineering and Management Sciences, 1(3), 122-129.

McCall, J.J. (1965). Maintenance policies for stochastically failing equipment: a survey. Management Science, 11(5), 493-524.

Mosman, A.B.A., Mostafa, S.A., Mustapha, A., Shamsudin, A.U., \& Ahmed, M. (2019). An optimized method for automated stadium attendance management system. International Journal of Engineering \& Technology, 8(1.7), 112-118.

Niwas, R., \& Garg, H. (2018). An approach for analyzing the reliability of an industrial system based on the cost free Warranty Policy. Journal of the Brazilian Society of Mechanical Sciences and Engineering, 40(5), 1-9.

Pierskalla, W.P., \& Voelker, J.A. (1976). A survey of maintenance models: the control and surveillance of deteriorating systems. Naval Research Logistics Quarterly, 23(3), 353-388.

Ram, M., \& Kumar, A. (2015). Performability analysis of a system under 1-out-of-2: G scheme with perfect reworking. Journal of the Brazilian Society of Mechanical Sciences and Engineering, 37(3), 1029-1038.

Ram, M., Singh, S.B., \& Singh, V.V. (2013). Stochastic analysis of a standby system with waiting repair strategy. IEEE Transactions on Systems, man, and cybernetics: Systems, 43(3), 698-707.

Shakuntla, S., Lal, A.K., Bhatia, S.S., \& Singh, J. (2011). Reliability analysis of polytube industry using supplementary variable technique. Applied Mathematics and Computation, 218(8), 3981-3992.

Sharma, S.P., \& Vishwakarma, Y. (2014). Application of Markov process in performance analysis of Markov feeding system of sugar industry. Journal of Industrial Mathematics, 2014, Article ID 593176.

Sherif, Y.S., \& Smith, M.L. (1981). Optimal maintenance models for systems subject to failure - a review. Naval Research Logistics Quarterly, 28(1), 47-74.

Srinath, L.S. (1994). Reliability engineering, $3^{\text {rd }}$ edition. East-West Press Pvt. Ltd., New Delhi, India.

Yusuf, I., Umar, M., \& Suleiman, K. (2018). Performance analysis of a single host system with three types of heterogeneous software. Research Journal of Applied Sciences, 13(12), 736-741. 
International Journal of Mathematical, Engineering and Management Sciences

Vol. 6, No. 1, 383-395, 2021

https://doi.org/10.33889/IJMEMS.2021.6.1.024

Zheng, J., Okamura, H., \& Dohi, T. (2018). Reliability importance of components in a realtime computing system with standby redundancy schemes. International Journal of Mathematical, Engineering and Management Sciences, 3(2), 64-89. 